\title{
Introduction to Ear and Temporal Bone Special Issue
}

\author{
Kelly R. Magliocca ${ }^{1}$ Michelle D. Williams ${ }^{2}$
}

Received: 1 May 2018 / Accepted: 8 May 2018 / Published online: 1 August 2018

c) Springer Science+Business Media, LLC, part of Springer Nature 2018

Not unlike the oral cavity and sinonasal tract, the region of the ear and temporal bone includes numerous tissue types in close proximity including skin, adnexal structures, cartilage, bone, synovial and mucosal linings, and mesenchymal elements. Consequently, a wide variety of disorders including infectious, inflammatory, autoimmune, benign and malignant neoplasms can be expected to affect the ear and temporal bone. The two commonly reported and principal methods of studying histologic tissues of the ear/temporal bone have first included the examination of biopsy material, which often is limited in size, given the anatomic location or the disease in question. Second, examining sections of cadaver ear and temporal bone has allowed evaluation of multiple anatomic sites and tissues in relation to one another and was key to early progress in understanding histopathologic changes.

More recently with the growth of specialized head and neck oncology centers, composite resections including the ear, temporal bone, and surrounding structures are increasingly encountered for advanced and often recurrent periauricular skin cancers, and T4 parotid gland tumors and has provided additional insight.

The understanding of ear and temporal bone pathology therefore requires appreciation of anatomic associations, radiologic findings, and surgical approaches to correlate with the spectrum of histologic alterations encountered. Moreover, the broad spectrum of diseases which may be encountered in this region must be considered for thorough differential diagnoses and correlation with both gross and microscopic findings. In this Special Issue of Head and Neck Pathology devoted to Ear and Temporal Bone Pathology, the authors detail a broad spectrum of disease entities encountered in this region, providing useful criteria supplemented with high-quality photomicrographs, and valuable illustrations to facilitate identification and reporting of otologic disease. It is hoped that the reviews presented herein will be practical and informative now and during those challenging future cases to be encountered.

Acknowledgements We sincerely thank all of the authors who have provided their time and expertise for this collection as well as Drs. Susan Muller and Lester Thompson for their patience and support.

\section{Compliance with Ethical Standards}

Conflict of interest The authors individually declare no conflicts of interest with regard to this submission.

Research Involving Human and Animals Participants This article does not contain any studies with human participants or animals performed by any of the authors.
Kelly R. Magliocca

kmagliocca@emory.edu

Michelle D. Williams

mdwillia@mdanderson.org

1 Department of Pathology and Lab Medicine, Emory University, 550 Peachtree St. NE, Atlanta, GA 30308, USA

2 Division of Pathology/Lab Medicine, Department of Pathology, The University of Texas MD Anderson Cancer Center, Houston, TX 77030, USA 